\title{
Validity of self blood pressure measurement in the control of the hypertensive patient: factors involved
}

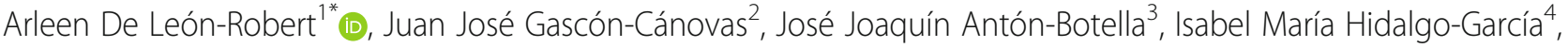 \\ Carmen López-Alegría ${ }^{5}$, Yoalys Dilvani Pérez-Cabrera ${ }^{6}$ and Heidy Merari Campusano-Castellanos ${ }^{7}$
}

\begin{abstract}
Background: Improving clinical practice aimed at controlling hypertension is a pending issue in health systems. One of the methods currently used for this purpose is self blood pressure measurement (SBPM) whose use increases every day. The aims of this study are to establish the optimal cut-off point for the 3-day SMBP protocol and to identify factors that could affect the precision of the 3-day SMBP protocol using 24-h ambulatory blood pressure monitoring (ABPM) as a reference.

Method: This is a cross-sectional descriptive study to validate a diagnostic test performed by a primary care team in Murcia, Spain. A total of 153 hypertensive patients under 80 years of age who met the inclusion criteria were evaluated. ABPM was performed for $24 \mathrm{~h}$. The SBPM protocol consisted of recording 2 measurements in the morning and 2 at night for 3 days.

Results: The cut-off point for SBP was set at $135 \mathrm{mmHg}$ (sensitivity: 80.39\%, specificity: 74.19\%), and for DBP, it was set at $83 \mathrm{mmHg}$ (sensitivity: 76.48\%, specificity: 84.89\%), which yielded the highest combined sensitivity and specificity. After carrying out the validation study with the new figures, we proceeded to establish which socio-demographic factors prevented a correct classification of patients. These errors were more common in male patients for the assessments of both $\mathrm{DBP}(\mathrm{OR}=2.4)$ and $\mathrm{SBP}(\mathrm{OR}=2.5)$; hypertensive patients with age $<67,5$ years $(\mathrm{OR}=1,5)$; having no work activity $(\mathrm{OR}=3,6)$ and with concomitant chronic kidney disease (CKD) $(\mathrm{OR}=5.0)$.
\end{abstract}

Conclusion: Being male, older than 67.5 years, with CKD or with no work activity increases the probability of being misclassified for hypertension during follow-up as assessed by SBPM over 3 days.

Trial registration: This study was approved by the research ethics committee of the University of Murcia under registration number 1018/2015.

Keywords: Hypertension, Self blood pressure, Primary care, Diagnostic errors

\section{Background}

A primary objective in any primary care consultation is to achieve good blood pressure (BP) control in hypertensive patients in order to reduce the likelihood of future cardiovascular events. Hypertension (HT) in Spain affects $36.7 \%$ of the total population, while approximately $40 \%$ of the general population in Europe is affected [1]. In both men and women, this prevalence increases significantly with age, reaching $68 \%$ of those over 60 year

* Correspondence: arleendo@yahoo.com

${ }^{1}$ Fortuna Health Centre, Murcia, Spain

Full list of author information is available at the end of the article
[2]. In terms of morbidity and mortality, HT has been associated with 1 in 2 deaths of cardiovascular origin in individuals older than 50 years [3].

Currently, SBPM is considered a useful tool both in the diagnosis and monitoring of hypertension $[1,4-6]$. Although it cannot replace ABPM as a gold-standard assessment method, it is considered a complementary method in the diagnosis of high blood pressure [7].

The main advantage of SBPM lies in its ability to obtain multiple BP records outside of the health care environment. Likewise, SBPM shows considerable agreement with $\mathrm{ABPM}$ in regard to detecting normotension and

(c) The Author(s). 2019 Open Access This article is distributed under the terms of the Creative Commons Attribution 4.0 International License (http://creativecommons.org/licenses/by/4.0/), which permits unrestricted use, distribution, and reproduction in any medium, provided you give appropriate credit to the original author(s) and the source, provide a link to the Creative Commons license, and indicate if changes were made. The Creative Commons Public Domain Dedication waiver (http://creativecommons.org/publicdomain/zero/1.0/) applies to the data made available in this article, unless otherwise stated. 
masked hypertension [4]. The SBPM's correlation with damage to target organs and its ability to predict cardiovascular events is higher than in-office blood pressure measurements [8] and is similar to that of ABPM [9]. Patients monitored with this technique show better adherence to long-term pharmacological treatments and therefore improved control rates, lower follow-up costs, better assessments of the effects of pharmacological treatments at different times of the day, the elimination of observer bias, and less clinical inertia [4].

There is no doubt that SBPM is a useful clinical tool for hypertensive patients in Primary Care, where the good management of the short time available for the consultation and the reliability are essential for appropriate control of HT. Therefore, it is also necessary to have reliable cut-off values and an efficient daily SBPM decision-making scheme. Therefore, after demonstrating that the 3-day protocol is the most efficient option for the control of blood pressure in primary care, with a discriminative capacity and agreement with ABPM similar to other protocols $[10,11]$, the aims of the present study are to establish the optimal cut-off point for the 3-day SBPM protocol and to identify the socio-demographic factors that can influence erroneous classification by SBPM during the monitoring of hypertensive patients.

\section{Methods}

This is a cross-sectional descriptive study for the validation of a diagnostic test.

\section{Population}

We worked with hypertensive patients registered in the computer system at the Vistalegre-La Flota Urban Health Centre in Murcia, Spain and those receiving routine care.

\section{Study period}

December 2011 to December 2012.

\section{Criteria for inclusion and exclusion}

Inclusion criteria included the diagnosis of complicated and uncomplicated hypertension, an age of 18-80 years, sufficient vision and hearing to perform the selfmeasurement, and adequate intellectual capacity to obtain the measurements or the oversight of a responsible caregiver for doing so. The exclusion criteria that were considered are those considered valid in various international HT guidelines $[1,5]$ : Immobilized patients without a responsible caregiver and hypertensive patients diagnosed with obsessive-compulsive disorder.

\section{Selection mechanism}

Duplicates, diagnostic errors, patients in the computerized clinical history, and those with their last visit to the health centre (to see a doctor or nurse) in the year prior to the start of the patient selection $(N=2,245)$ were filtered from anonymized lists. The sample was calculated with an accuracy of 5\%, a confidence level of $95 \%$, and a sensitivity and specificity of 85.7 and $75 \%$, respectively. An estimated prevalence of patients with uncontrolled BP of $35 \%(n=141)$ was determined. From this figure, we calculated a percentage of expected losses of $15 \%$, leading to a sample size of $n=153$. The calculation of the sample size was performed using the online calculator by Fisterra [12].

\section{Sampling method}

A systematic random sampling procedure was carried out. The first subject was chosen at random, and the sampling fraction used was $1 / 10$ patients. The patients were recruited through telephone contact or through their doctors, for those who went to the health centre during the patient selection period. In the case of negative or no contact after several attempts, the next patient was chosen from the list. The recruitment flowchart is shown in Fig. 1.

After giving consent, each selected patient who agreed to participate was scheduled for an appointment at 8:30 a.m. to perform the ABPM. The perimeter of the patient's arm was measured, and the appropriate cuff was provided. If the arm circumference was greater than 32 $\mathrm{cm}$, a large cuff was provided. The blood pressure was measured in both arms, and the non-dominant arm was chosen as the measuring arm. If both measurements were equal, the cuff was placed on the left arm for righthanded patients or on the right arm for left-handed patients. After the placement of the ABPM device, a forced measurement was immediately carried out. The recording began in the morning at the time the device was placed.

The patients were instructed to perform normal daily activities, except that when the cuff warned that a measurement was about to begin, the arm should be kept in a relaxed position. The patients were asked to return the next day at the same time.

The programming of the device was as follows: Frequency of readings: every $15 \mathrm{~min}$ during daytime and every $30 \mathrm{~min}$ while sleeping. For this, the patient was questioned when placing the device about their sleep schedule, which was confirmed and readjusted the next day when the device was removed. With these data, the program calculated the beginning of the night or sleep period and the day or activity period for the purposes of the analysis. Types of recordings: Measurement of SBP, DBP and HR over $24 \mathrm{~h}$, daytime and evening. Measurement range: HR: 40 to 180 beats per minute. Pressure: 70 to $285 \mathrm{mmHg}$ for systolic; 40 to $200 \mathrm{mmHg}$ for diastolic and 60 to $240 \mathrm{mmHg}$ for mean blood pressure 


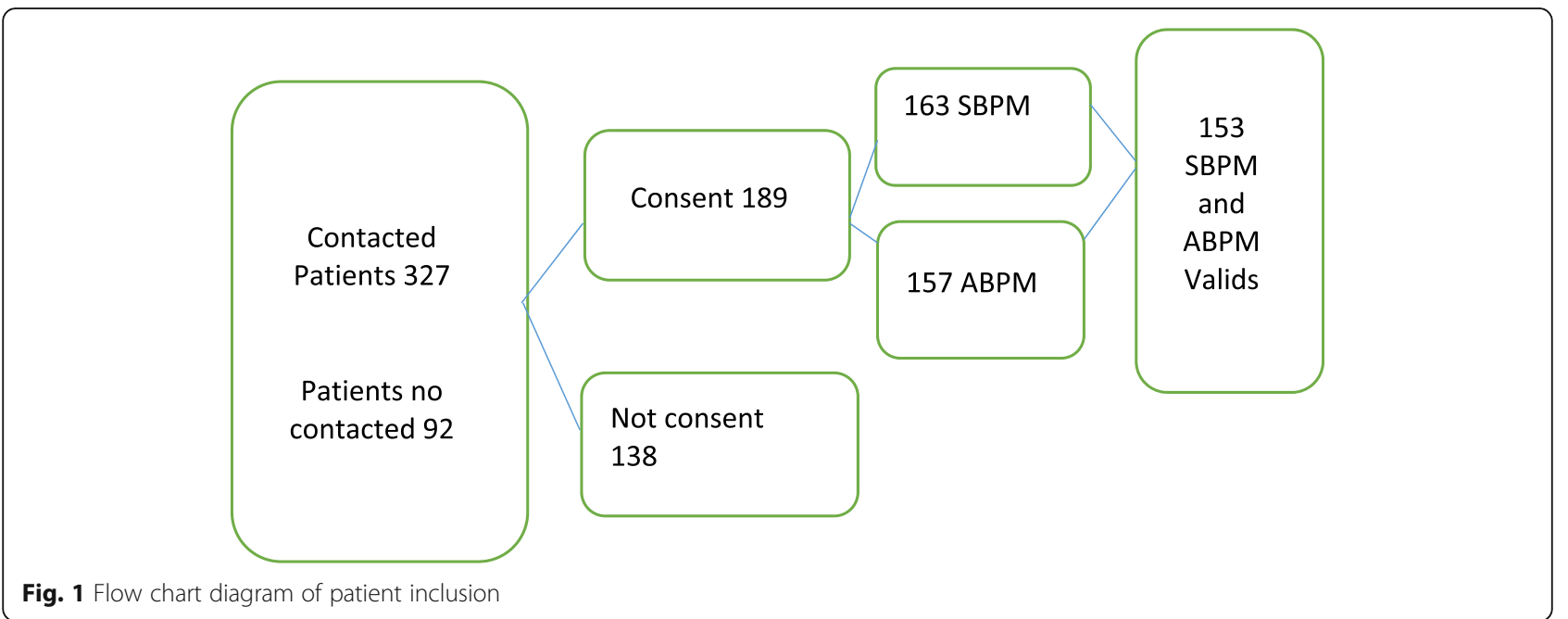

values. Valid record criteria: $70 \%$ valid measurements on the ABPM, more than 14 valid measurements of systolic and diastolic BPs during the day and more than 7 measurements of systolic and diastolic BPs during the night.

The next day, the ABPM device was removed and the patient was shown how to use the validated semi-automatic tensiometer to obtain SBPM records. The subject was seated, and after a period of $5 \mathrm{~min}$ of rest, 2 measurements were taken from the dominant arm with a 1-min interval between them. A third measurement was taken if the first 2 had a difference of greater than $5 \mathrm{mmHg}$.

After the demonstration, the patient was instructed in the proper handling of the device, specifically that the measurements should be taken while sitting, at rest, with the cuff placed on the arm that showed the highest BP. The patient was also instructed on how to place the cuff, which was to be $2-3 \mathrm{~cm}$ above the flexure of the elbow, its fit on the arm, and the position at which to take the pressure reading.

The correct performance of the first 2 measurements on the first day was verified by the patient in front of the researcher. Then, the blood pressure monitor was given to the patient, who was instructed in writing to take 2 measurements in the morning (between 7:00 and 10:00 a.m.) and 2 before going to bed (between 21:00 and 23:00 p.m.) with 1-2 min between measurements for 3 consecutive days. A total of 12 measurements were obtained for each patient (the first day measurements were later discarded), establishing the 3-day SBPM pattern. The instruments used in this study included 2 ABPM devices Microlife Watch BP 03 (Microlife, Widnau, Switzerland) [13] and 10 automatic arm blood pressure monitors for SMBP (Microlife Watchbp Home) that were validated according to the standards of the Spanish Hypertension Society (SEH) and the British Hypertension Society (BHSOC) (accessible on January 11, 2016 at: https://www.seh-lelha.org/microlife-watchbp-o3/ and https://bihsoc.org/bp-monitors/for-home-use/).
Regarding the definitions of the variables applied in this study, we defined poor HT control as when the mean BP measured by ABPM over $24 \mathrm{~h}$ was greater than $130 / 80 \mathrm{mmHg}$. For the 3-day SMBP, we considered uncontrolled average blood pressure as greater than $135 / 85 \mathrm{mmHg}$.

This descriptive study was carried out with the demographic variables of age, sex and work status, taking into account the records of spanish administrative document (TSI) that accredits access to citizens to health care benefits. If the patient was not active: TSI $<002$ (Without income, retired) or active: TSI 003 (income level $<€ 18,000 /$ year); TSI $>4$ (income level $>€ 18,000 /$ year). Clinical variables included associated comorbidities such as a diagnoses of dyslipidaemia, diabetes mellitus, chronic kidney disease, atrial fibrillation, and stroke.

\section{Statistical analysis}

The statistical programs SPSS version 22 and Epidat version 3.1 were used to analyse the data.

Categorical variables are presented as absolute frequencies (\%) and quantitative variables as the means and standard deviations. Values of $p<0.05$ were considered statistically significant, and $95 \%$ confidence intervals $(95 \% \mathrm{CI})$ were calculated. The sources of the data were the BP records collected by SBPM and ABPM as well as the electronic health records of the patients in the OMI-AP program.

\section{Test validation analysis}

A comparison of the data collected using the 3-day SBPM (discarding measurements from the first day) was performed using the figures obtained by the 24-h ABPM as the reference standard. Given the results obtained by De León-Robert et al. [10], the 3-day pattern was chosen (SBPM-DAYS-2\&3) and an optimal cut-off point was obtained. The calculations of the predictive capacity of 
the 3-day protocol with the new cut-off point were performed using a statistical calculator. With the data obtained, the cases with misclassifications (false positives and negatives) in the SBPM-DAYS-2\&3 data were identified at the cut-off point where the sensitivity and specificity of the diagnosis of HT were optimized, using the $24-\mathrm{h}$ ABPM as the gold standard $(130 / 80 \mathrm{mmHg})$. An analysis of how sociodemographic and clinical factors related to errors in diagnostic classifications was performed. Calculations were performed for the crude and adjusted odds ratios, according to sex, of the diagnostic classification errors for both systolic and diastolic HT. These were performed using logistic regression models that included age, income level, and clinical comorbidities such as diabetes, dyslipidaemia, chronic kidney disease, ictus, and atrial fibrillation as predictor variables. The estimating of the agreement between ABPM and SBPM readings were perfomed using the Bland-Altman plot analysis and the concordance was determinated using intraclass correlation coefficient (ICC).

\section{Results}

A total of 419 patients were selected for inclusion in the analysis, of whom 153 completed SBPM and ABPM with the required quality (Fig. 1). Of these patients, 50.3\% were women. The mean age was 61.54 with a range of $23-80$ years. In terms of economic activity, $67.3 \%$ were classified as having no work activity (pensioners and the unemployed) (Table 1).

In addition to HT, other associated chronic pathologies were found among the hypertensive patients studied (Table 1). The most common was dyslipidaemia (41.8\%).
According to the results of the ROC curve, the cut-off point where sensitivity and specificity were optimized was $135.5 / 83 \mathrm{mmHg}$ for the diagnosis of uncontrolled HT. The mean differences between the two methods (ABPM and SBPM) are 10 and $5 \mathrm{mmHg}$ for systolic and diastolic pressure respectively (Fig. 2). The concordance between SBPM and ABPM according ICC was higher for diastolyc pressure $(0,64 ; p<0.001)$ than for systolic pressure $(0,44 ; p<0.001)$.

Based on this cut-off point, the sensitivity of the SBPM-DAYS-2\&3 test for identifying cases with control problems in some BP measurements was $87.7 \%$, while its specificity was notably lower $(62.5 \%)$ (Table 2$)$. More specifically, this test was more sensitive for the isolated diagnosis of abnormal SBP values (87.7\%) than for diagnosing abnormal DBP values (70.6\%); In contrast, it had a greater specificity when the uncontrolled blood pressure was diastolic $(85.7 \%)$ instead of systolic $(74.2 \%)$ (Table 2). On the other hand, the predictive capacity of the test for the diagnosis of a lack of control of systolic or diastolic values, with a prevalence in the study sample of $42.5 \%$, was elevated for a negative result $(87.3 \%)$ and moderate for a positive result (63.35\%) (Table 2).

With the SBPM-DAYS-2\&3, approximately 1 out of every 4 hypertensive subjects had their systolic HT incorrectly classified (false positive or negative); this figure dropped to 1 out of every 6 for the assessment of diastolic HT. The variables associated with obtaining an erroneous result with the SBPM-DAYS-2\&3 test were sex, age, work status and chronic kidney disease. These errors were approximately 2.5 times more common in male patients for the assessments of both DBP (OR = $2.4)$ and SBP $(\mathrm{OR}=2.5)$. Regarding the latter, in subjects

Table 1 Socio-demographic and baseline clinical characteristics of the study subjects

\begin{tabular}{|c|c|c|}
\hline Gender & $\mathrm{n}$ & $\%$ \\
\hline Male & 76 & 49.7 \\
\hline Female & 77 & 50.3 \\
\hline \multicolumn{3}{|l|}{ Age group } \\
\hline (23-57.5 years) & 50 & 32.7 \\
\hline (57.51-67.5 years) & 52 & 34.0 \\
\hline (> 67.5 years) & 51 & 33.3 \\
\hline \multicolumn{3}{|l|}{ TSI } \\
\hline Inactive (TSI 1 Unemployed; TSI 2 Retired) & 103 & 67.3 \\
\hline Active (TSI 3 Income below $€$ 18,000/year; TSI 4 Income above $€$ 18,000/year) & 50 & 32.7 \\
\hline \multicolumn{3}{|l|}{ Comorbidities } \\
\hline Diabetes & 41 & 26.8 \\
\hline Chronic kidney disease & 13 & 8.5 \\
\hline Dyslipidaemia & 64 & 41.8 \\
\hline Atrial fibrillation & 7 & 4.6 \\
\hline
\end{tabular}

$\mathrm{n}$ : absolute frequency. \%: relative frequency 


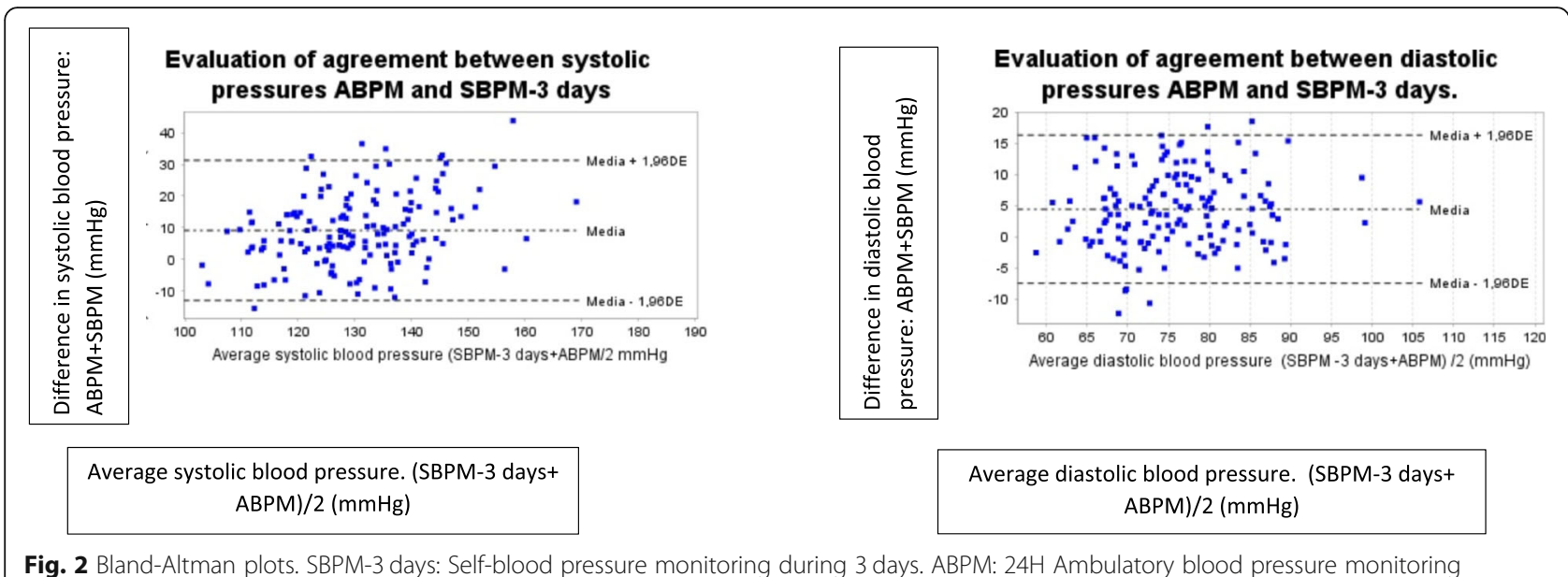

Fig. 2 Bland-Altman plots. SBPM-3 days: Self-blood pressure monitoring during 3 days. ABPM: 24H Ambulatory blood pressure monitoring

of more advanced ages ( $>67.5$ years), the validity of the test was lower $(\mathrm{OR}=1.5)$. The same pattern occurred in patients with TSI $\leq 3$ (low income level) $(\mathrm{OR}=3.6)$ and in hypertensive patients with concomitant chronic kidney disease $(\mathrm{OR}=5.0)$ (Table 3$)$.

\section{Discussion}

The diagnostic capacity of SBPM, using ABPM as a reference, for the assessment of HT has been widely investigated by several studies [14]. The possibility of using its different protocols without diminishing that capacity has also been investigated, with good results [15]. Taking into account the data obtained to date, as well as the need in primary care for an efficient protocol, we decided to evaluate the SBPM-DAYS-2\&3. We also investigated which factors we should take into account for its correct application and interpretation. The analysis with ROC curves for the 3-day protocol resulted in cut-off values of 135.5 $\mathrm{mmHg}$ for SBP and $83 \mathrm{mmHg}$ for DBP, differing from the results of another study [11] by $3 \mathrm{mmHg}$ for SBP and just $0.2 \mathrm{mmHg}$ for DBP. However, the SBPM-DAYS-2\&3 yields higher values for both sensitivity and specificity for both pressures with our cut-off values.
The current guidelines on hypertension by various societies do not provide clear recommendations in their protocols about all of the factors that can diminish the accuracy of SBPM as a follow-up method in HT. The Japanese and Brazilian guidelines refer to the need for bladder emptying before taking BP measurements, something not mentioned in the European or American guidelines [16]. For Almeida et al. [16], who also used the 3-day protocol, a factor that limits the accuracy of SBPM is whether BP measurements are taken after urination. Other studies, such as ours, note that sex and age are possible factors. However, they also add the systolic and diastolic figures, the number of readings, food intake, alcohol consumption, the taking of BP measurements after bathing, and being a smoker [14, 17-19]. However, some of those studies accounted for the daytime ABPM to make their comparisons $[16,19]$.

Among the clinical factors evaluated by our study, it is worth mentioning the significant influence of the diagnosis of chronic kidney disease in the sample as a factor involved in limiting the accuracy of the SBPM-DAYS$2 \& 3$ protocol. Studies in kidney patients indicate greater arterial rigidity in these patients, which could be the cause. Although arteriosclerosis is also common among

Table 2 Validation of self-monitoring of blood pressure for 3 days (cut-off point $=135.5 / 83 \mathrm{mmHg}$ ) for the control of hypertension. Gold standard: 24-h ambulatory blood pressure monitoring

\begin{tabular}{|c|c|c|c|c|c|c|c|c|c|}
\hline \multirow{3}{*}{ Sensitivity (\%) } & \multicolumn{3}{|c|}{ Systolic hypertension } & \multicolumn{3}{|c|}{ Diastolic hypertension } & \multicolumn{3}{|c|}{ Joint HT } \\
\hline & \multirow{2}{*}{$\begin{array}{l}\mathrm{Ep} \\
80.4\end{array}$} & \multicolumn{2}{|c|}{$95 \% \mathrm{Cl}$} & \multirow{2}{*}{$\begin{array}{l}\mathrm{Ep} \\
70.6\end{array}$} & \multicolumn{2}{|c|}{$95 \% \mathrm{Cl}$} & \multirow{2}{*}{$\begin{array}{l}\mathrm{Ep} \\
87.7\end{array}$} & \multicolumn{2}{|l|}{$95 \% \mathrm{Cl}$} \\
\hline & & 69.1 & 91.7 & & 53.8 & 87.3 & & 79.0 & 96.4 \\
\hline Specificity (\%) & 74.2 & 65.0 & 83.4 & 85.7 & 79.0 & 92.4 & 62.5 & 51.8 & 73.2 \\
\hline Positive predictive value (\%) & 64.3 & 52.4 & 76.2 & 58.5 & 42.2 & 74.8 & 63.3 & 52.8 & 73.8 \\
\hline Negative predictive value (\%) & 86.7 & 78.8 & 94.6 & 91.1 & 85.3 & 96.8 & 87.3 & 78.3 & 96.3 \\
\hline Positive likelihood ratio & 3.1 & 2.2 & 6.5 & 4.9 & 1.7 & 4.9 & 2.3 & 2.6 & 9.91 \\
\hline Negative likelihood ratio & 0.3 & 0.22 & 0.46 & 0.3 & 0.1 & 0.3 & 0.2 & 0.3 & 0.6 \\
\hline
\end{tabular}

Prevalence of uncontrolled status: $42.5 \%$

Ep point estimate, $H T$ hypertension, \% percentage, $95 \%$ Cl 95\% confidence interval 
Table 3 Factors that influence the misclassification of hypertension by SBPM. Gold standard: ABPM (130/80 mmHg). SBPM test 1 on 3rd day: (135.5/83 mmHg)

\begin{tabular}{|c|c|c|c|c|}
\hline & SMBP SBP & & SMBP DBP & \\
\hline & OR (95\%Cl) & $\overline{\text { Adjusted } \mathrm{OR}^{\mathrm{a}}}$ & $\overline{\mathrm{OR}(95 \% \mathrm{Cl})}$ & Adjusted $\mathrm{OR}^{\mathrm{C}}$ \\
\hline Male & $2.1(0.99-4.5)$ & $2.5(1.1-5.5)^{*}$ & $2.3(1.0-5.5)$ & $2.4(1.0-5.9)^{*}$ \\
\hline Age $>67.5$ years & $1.5(1.0-2.2)^{*}$ & $1.5(1.0-2.3)^{*}$ & $1.2(0.8-1.9)$ & $1.2(0.8-1.9)$ \\
\hline $\mathrm{TS} \mid \leq 3$ & $2.9(0.8-10.0)$ & $3.6(1.0-12.5)^{*}$ & $1.4(0.5-3.9)$ & $1.2(0.4-3.4)$ \\
\hline Diabetes & $1.3(0.6-2.8)$ & $2.1(1-4.5)$ & $1.1(0.9-2.1)$ & $1.1(0.9-2.3)$ \\
\hline IRC & $6.4(2-21.2)^{*}$ & $5(1.9-20)^{*}$ & $2.3(0.9-8.3)$ & $1.9(0.9-8.3)$ \\
\hline Dyslipidaemia & $1.1(0.9-1.8)$ & $1.1(0.9-1.9)$ & $1.1(0.9-2)$ & $1.1(0.9-2)$ \\
\hline Fibrillation & $1.3(0.9-7.1)$ & $1.1(0.9-5.5)$ & $1.3(0.9-11.2)$ & $1.8(0.9-15.6)$ \\
\hline
\end{tabular}

HT High blood pressure, SBPM Self-monitoring of blood pressure, ABPM Ambulatory monitoring of blood pressure, SBP Systolic blood pressure, $D B P$ Diastolic blood pressure, OR Odds ratio, $T S I<4$ unemployed, retired, income less than $€ 18,000,95 \% C / 95 \%$ confidence interval, $C K D$ Chronic kidney disease ${ }^{*} p<0.05$. ${ }^{* * *} p<0.01{ }^{\text {a Adjusted }}$ for sex by logistic regression using the ENTER method

diabetic patients, no significant difference was found between diabetic and non-diabetic patients.

\section{Limitations of the study}

This study was performed with a small sample size in a single health centre in Spain, wich is not necessarily representative of the general population. However, as a strength, the study was conducted in the field of usual clinical practice.

\section{Conclusions}

The cut-off point with the highest joint sensitivity and specificity for the SMBP-DAYS-2\&3 protocol is 135.5/ $83 \mathrm{mmHg}$. Being male, over 67.5 years of age, having low income level, and having a diagnosis of chronic kidney disease can all reduce the validity of the SMBP-DAYS$2 \& 3$ protocol for classifying hypertensive patients as poorly controlled during follow-up.

\section{Abbreviations}

ABPM: Ambulatory blood pressure monitoring for 24 h; DBP: Diastolic blood pressure; HR: Heart rate; HT: Hypertension; IC95\%: 95\% confidence interval; ROC CURVES: Receiver operating characteristic curves.; SBP: Systolic blood pressure; SMBP: Self-monitoring of blood pressure; SMBP-DAYS-2\&3: 3-day protocol of self-monitoring of blood pressure; TSI: Individual health card

\section{Acknowledgements}

We thank the patients and the physicians at the Vistalegre-La Flota Health Centre for their collaboration.

\section{Authors' contributions}

All of the authors participated in the conception of the study. The bibliographic reviews were made by $I H G, C L A, Y D P C, J J A B$ and ADR. The study design and analysis were carried out by JJGC. The field work was performed by CLA, YDPC, IMHG and ADLR. The successive versions of the manuscript were written by ADLR and JJGC. All of the authors participated in the review of the article with important contributions and accepted the final version.

\section{Funding}

This research work has been partially funded through a research grant from Cajamurcia with the file number FFIS/CM10/031.

\section{Availability of data and materials}

The datasets generated and/or analysed during the current study are not publicly available due to data privacy laws but are available from the corresponding author on reasonable request.

Ethics approval and consent to participate

This study was approved by the research ethics committee of the University of Murcia under registration number 1018/2015, after which, the consent of the patients was obtained verbally, after a verbal report of the procedures to be performed.

\section{Consent for publication}

Not applicable.

\section{Competing interests}

The authors declare that they have no competing interests.

\section{Author details}

${ }^{1}$ Fortuna Health Centre, Murcia, Spain. ${ }^{2}$ Faculty of Medicine (University of Murcia) - Instituto Murciano de Biosanitaria - Arrixaca (IMIB-Arrixaca), Murcia, Spain. ${ }^{3}$ Murcia Health Centre, Murcia, Spain. ${ }^{4}$ Vistalegre-La Flota Health Centre, Murcia, Spain. ${ }^{5}$ Cieza Este Health Centre, Murcia, Spain. ${ }^{6}$ Morella Health Centre, Castellón, Spain. ${ }^{7}$ Abanilla Health Centre, Murcia, Spain.

Received: 15 May 2018 Accepted: 30 June 2019

Published online: 17 July 2019

\section{References}

1. Mancia G, Fagard R, Narkiewicz K, et al. 2013 ESH/ESC guidelines for the management of arterial hypertension: the task force for the management of arterial hypertension of the European Society of Hypertension (ESH) and of the European Society of Cardiology (ESC). Eur Heart J. 2013;34(28):2159-219.

2. Marín R, de la Sierra Á, Armario P, Campo C, Banegas JR, Gorostidi M. Guía sobre el diagnóstico y tratamiento de la hipertensión arterial en España 2005. Med Clin (Barc). 2005;125(1):24-34. https://doi.org/10.1157/13076402.

3. Graciani A, Clemencia Zuluaga-Zuluaga M, Banegas JR, María León-Muñoz L, de la Cruz JJ, Rodríguez-Artalejo F. Mortalidad cardiovascular atribuible a la presión arterial elevada en la población española de 50 años o más. Med Clin (Barc). 2008;131(4):125-9. https://doi.org/10.1157/13124098.

4. Divisón Garrote J, AMPA de la SEH-LELHA G. Medidas domiciliarias de presión arterial. Documento de consenso. SEH-LELHA 2014 Home blood pressure measurements. SEH-LELHA 2014 consensus documents. Hipertensión y Riesgo Vascular. 2015;32(1):27-39. https://doi.org/10.1016/j. hipert.2014.10.001

5. National Institute for Health and Clinical Excellence. NICE clinical guideline 127. Hypertension: clinical management of primary hypertension in adults. London: National Institute for Health and Clinical Excellence; 2011. [Accessed March, 2015]. Available from: http://www.nice.org.uk/nicemedia/ live/13561/56008/56008.pdf. 
6. Muxfeldt ES, Barros GS, Viegas BB, Carlos FO, Salles GF. Is home blood pressure monitoring useful in the Management of Patients with Resistant Hypertension? Am J Hypertens. 2015;28(2):190-9. https://doi.org/10.1093/ ajh/hpu145.

7. Di Monaco S, Rabbia F, Covella M, et al. Evaluation of a short home blood pressure measurement in an outpatient population of hypertensives. Clin Exp Hypertens. 2016. https://doi.org/10.1080/10641963.2016.1200600.

8. Stergiou GS, Siontis KC, lonannidis J. Home blood pressure as a cardiovascular outcome predictor: it's time to take this method seriously. Hypertension. 2010;55:1301-3.

9. Bliziotis IA, Destounis A, Stergiou G. Home Vs. ambulatory and office blood pressure in predicting target organ damage in hypertension: a systematic review and meta-analysis. Hypertens. 2012;30:1289-99.

10. De León-Robert A, Hidalgo-García I, Gascón-Cánovas J, Antón-Botella J, López-Alegría C, Campusano Castellanos H. Efficiency between the different measurement patterns of home blood pressure monitoring in the follow-up of hypertensive patients in primary care. Aten Primaria. 2019;51(4). https:// doi.org/10.1016/j.aprim.2017.11.006.

11. Kim J, et al. Measurements for the diagnosis of arterial hypertension: a prospective, multicenter study [abstract]. J Hypertens. 2015;33:e38.

12. Pita-Fernandez S. Determinación del tamaño muestral. 3. https://www. fisterra.com/mbe/investiga/9muestras/9muestras2.asp. Published 1996. Accessed 5 May 2016.

13. Stergiou GS, Tzamouranis D, Nasothimiou EG, Karpettas N, Protogerou A. Are there really differences between home and daytime ambulatory blood pressure? Comparison using a novel dual-mode ambulatory and home monitor. J Hum Hypertens. 2010;24(3):207-12. https://doi.org/10.1038/jhh.2009.60.

14. Stergiou GS, Bliziotis IA. Home blood pressure monitoring in the diagnosis and treatment of hypertension: a systematic review. Am J Hypertens. 2011; 24(2):123-34. https://doi.org/10.1038/ajh.2010.194.

15. Stergiou GS, Ntineri A. The optimal schedule for self-home blood pressure monitoring. J Hypertens. 2015;33(4):693-7. https://doi.org/10.1097/HJH. 0000000000000509 .

16. de AAEM, Stein R, Gus M, et al. Relevance to home blood pressure monitoring protocol of blood pressure measurements taken before firstmorning micturition and in the afternoon. Arq Bras Cardiol. 2014;103(4): 338-47. https://doi.org/10.5935/ABC.20140139.

17. Ito K, Obara T, Ohkubo T, et al. Influence of home blood pressure measuring conditions in the evening on the morning-evening home blood pressure difference in treated hypertensive patients: the J-HOME study. Blood Press Monit. 2009;14(4):160-5. https://doi.org/10.1097/MBP.0b013e32 832e $2 \mathrm{a} 40$

18. ISHIKAWA J, KARIO K, HOSHIDE S, et al. Determinants of exaggerated difference in morning and evening blood pressure measured by selfmeasured blood pressure monitoring in medicated hypertensive patients: Jichi morning hypertension research (J-MORE) study. Am J Hypertens. 2005; 18(7):958-65. https://doi.org/10.1016/j.amjhyper.2005.01.013.

19. Stergiou GS, Ntineri A, Kollias A, Destounis A, Nasothimiou E, Roussias L. Changing relationship among clinic, home, and ambulatory blood pressure with increasing age. J Am Soc Hypertens. 2015;9(7):544-52. https://doi.org/1 0.1016/j.jash.2015.04.002.

\section{Publisher's Note}

Springer Nature remains neutral with regard to jurisdictional claims in published maps and institutional affiliations.

Ready to submit your research? Choose BMC and benefit from:

- fast, convenient online submission

- thorough peer review by experienced researchers in your field

- rapid publication on acceptance

- support for research data, including large and complex data types

- gold Open Access which fosters wider collaboration and increased citations

- maximum visibility for your research: over $100 \mathrm{M}$ website views per year

At BMC, research is always in progress.

Learn more biomedcentral.com/submissions 